\title{
A Method to Measure Eye-Hand Coordination for Extracting Skilled Elements-Simultaneous Measurement of Eye-Gaze and Hand Location-
}

\author{
Atsuo Murata and Kosuke Inoue \\ Department of Intelligent Mechanical Systems, Division of Industrial Innovation Sciences, Graduate School of Natural Science and \\ Technology, Okayama University, Okayama 700-8530, Japan
}

\begin{abstract}
The purpose of this study was to construct bases for exploring the process of skill acquisition from the viewpoint of eye-hand coordination. The information obtained from eye-gaze is closely related to physical movements in any activity. It is important to establish a method to measure eye-hand coordination for extracting skilled elements and understanding the skill acquisition process. Using a system which consists of an eye mark recorder and a three-dimensional location measurement device, a method for measuring eye-hand coordination was proposed on the basis of the simultaneous measurement of eye-gaze and brush tip locations. After describing the measurement algorithm, the eye-hand coordination during calligraphy was exemplified. More concretely, using such a system, an attempt was made to show that the relationship between the line of eye-gaze and the brush tip stroke was different between a novice and an expert. In such a way, we suggested that the proposed method is promising for exploring the process of skill acquisition.
\end{abstract}

Key words: Skill, eye-hand coordination, eye-gaze, brush tip location, algorithm for measuring eye-hand coordination.

\section{Introduction}

An analysis of skill acquisition process is very important in the field of cognitive and movement sciences [1-5]. In a variety of tasks, the information obtained by visual information processing is essential for the determination and the execution of physical movements. It is assumed that the relationship between the eye movement and the physical movement differs according to the skill level. In order to learn and master the traditional skills efficiently, the research focusing on the cognitive processes and motor process is necessary and indispensable. Therefore, the exploration of the difference of eye-hand coordination between skilled and non-skilled participants would contribute to the realization of effective tradition method of skill.

Corresponding author: Atsuo Murata, Ph.D., professor, research fields: ergonomics, cognitive science. E-mail: murata@iims.sys.okayama-u.ac.jp.
Vickers [1] clarified different features of eye-hand coordination between skilled and non-skilled free-throw shooters. Murata et al. [2-4] suggested that the ability of eye-hand coordination and the ability of recognizing and predicting the work area are important elements in skilled tasks. Sano and Ukida [5], applying image processing techniques to the evaluation of characters in calligraphy, showed that the writing of characteristic components of calligraphy differed between skilled and non-skilled participants. In Ref. [2-4], the simultaneous measurement technique of eye-gaze and hand location is halfway, and is not systematically described so that a similar measurement system of eye-hand coordination can be made easily and with high measurement accuracy.

Therefore, a system that can simultaneously measure the eye-gaze and the brush tip locations has been developed, and its algorithm has been systematically described. The purpose of this study 
was to construct bases for clarifying the process of skill acquisition from the viewpoint of eye-hand coordination. The paper consists of the following steps. First (in Section 2.1), we explained apparatus for measuring the eye-hand coordination, that is, an eye mark recorder and a three-dimensional magnetic movement tracking system. Second (Sections 2.2-2.4), a method for measuring eye-hand coordination was proposed and fully described. This method consisted of the following three processes: (1) calculation of pen tip location (Section 2.2), (2) calculation of eye-gaze location after correction of binocular parallax (Section 2.3), and (3) transformation of eye-gaze location to the same coordination system with pen tip location (Section 2.4). Third (in Section 3), calligraphy was selected as a demonstrative application of simultaneous measurements of eye-gaze and brush tip location.

\section{Method for Measuring Eye-Hand Coordination}

\subsection{Apparatus}

The system consisted of a wearable type eye mark recorder (Nac Image Technology, EMR-9) (Fig. 1) and a three-dimensional magnetic motion tracker (Polhemus, FASTRAK) (Fig. 2). Both eye mark location and brush tip location were sampled with a sampling frequency of $60 \mathrm{~Hz}$. The eye mark recorder EMR-9 consists of a head unit equipped with a view camera and a camera for measuring line of gaze, and is not influenced by head sway. FASTRAK can obtain relative location between the transmitter and Receivers 1 or 2 with an accuracy of $0.8 \mathrm{~mm}$, if Receiver 1 or 2 is located within $760 \mathrm{~mm}$ from the transmitter. This system can measure the coordinates $X, Y$, and $Z$ and yaw $\varphi_{y}$ (rotation angle along $Z$-axis), pitch $\varphi_{p}$ (rotation angle along $Y$-axis), and roll $\varphi_{r}$ (rotation angle along $X$-axis) on the transmitter coordination system. In order to restrain the effects of magnetic field on the measurement as much as possible, we used a desk and a chair for calligraphy which are made of wood.

In Fig. 3a, Receiver 1 of FASTRAK is attached to a brush, and in Fig. 3b, Receiver 2 of FASTRAK is attached to EMR-9. Fig. 4 corresponds to the camera screen coordination system viewed from a view camera. The eye-gaze points after the correction of view difference between right and left eyes were used as a representative of eye location. The overview of measurement system and coordination systems (transmitter coordination system, Receiver 1 coordination system, Receiver 2 coordination system, view camera coordination system, and camera screen coordination system) is shown in Fig. 5. In the eye-hand coordination measurement algorithm, these coordination systems must be integrated to a common coordination system. The algorithm is mentioned below.

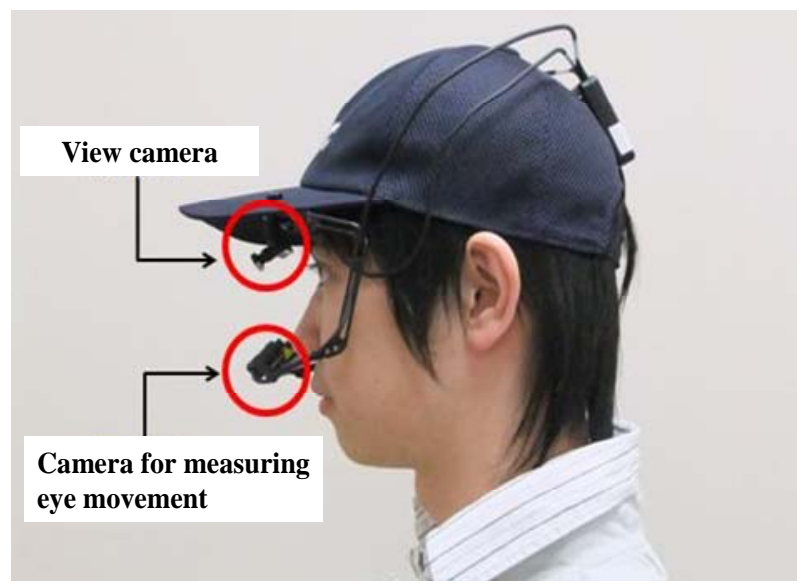

Fig. 1 View camera and camera for measuring eye movement (EMR-9).

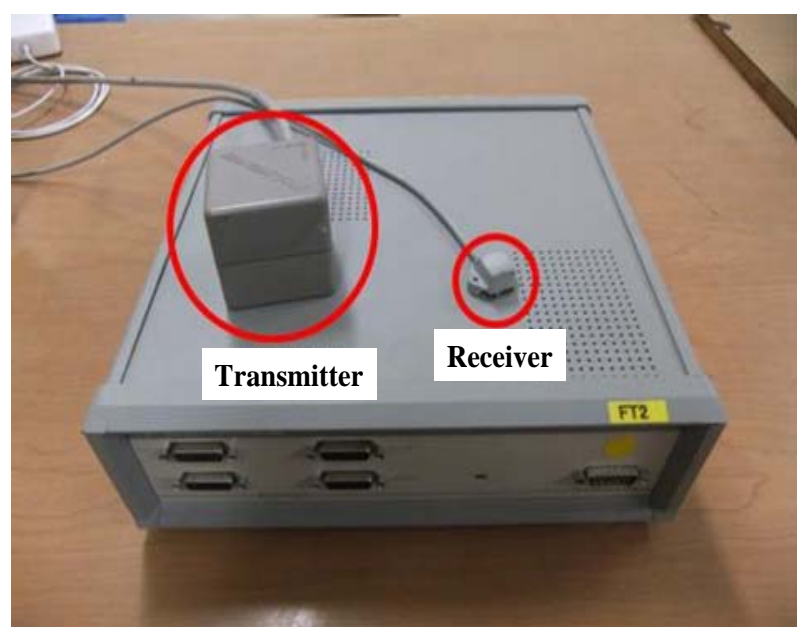

Fig. 2 FASTRAK, transmitter, and receiver for measuring three-dimensional locations. 


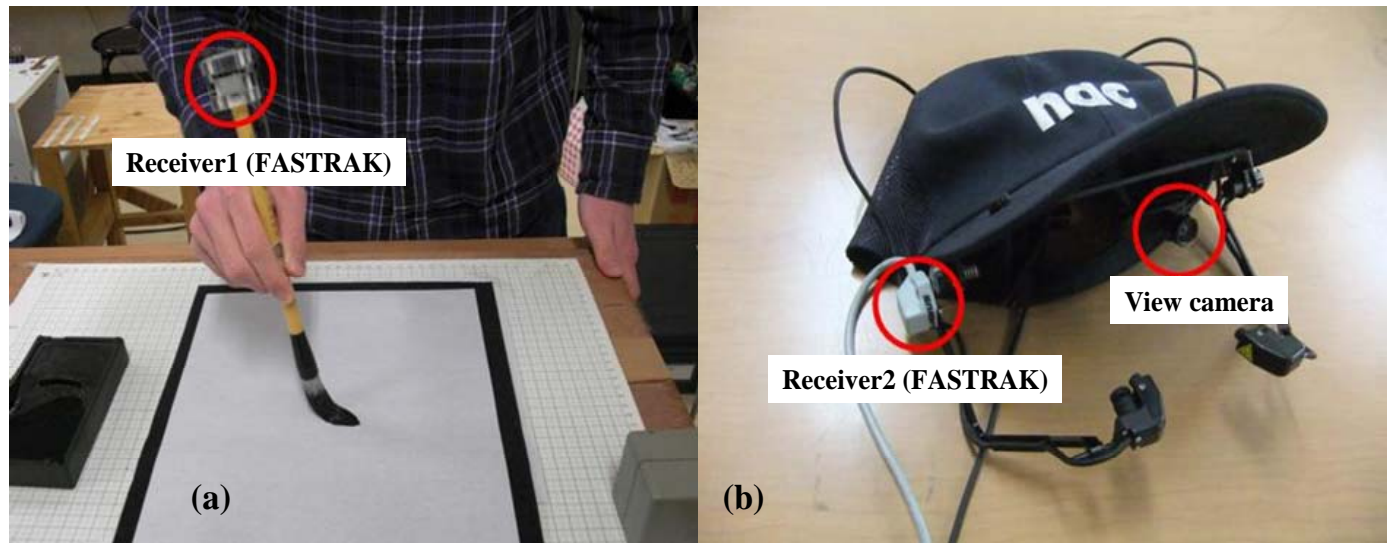

Fig. 3 (a) Attachment of Receiver 1 of FASTRAK to a brush; and (b) attachment of Receiver 2 of FASTRAK to EMR-9.

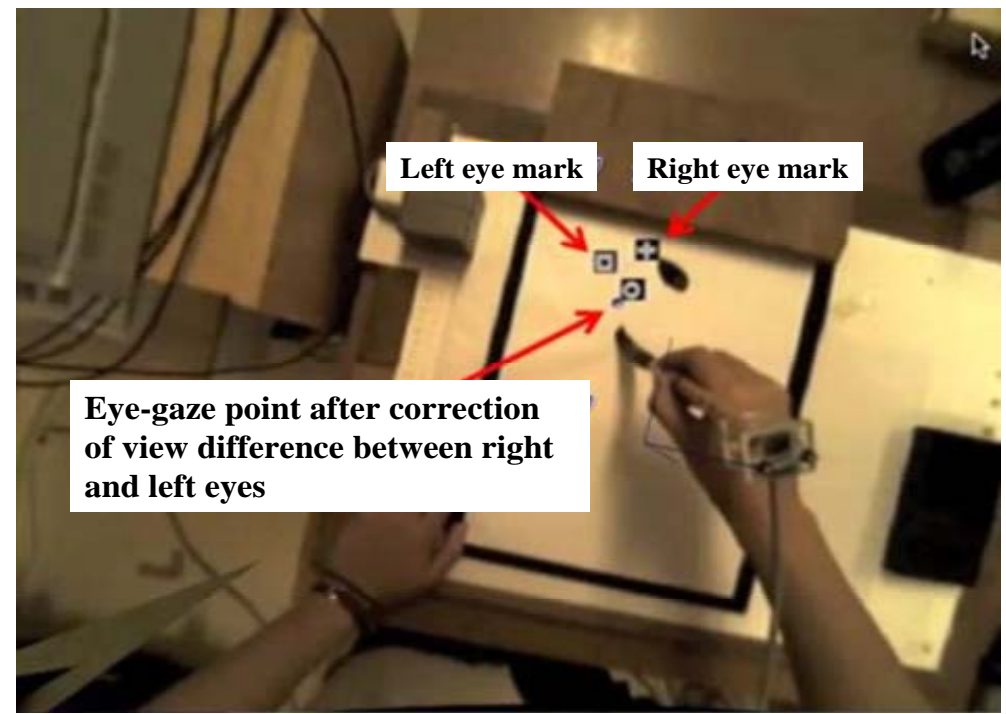

Fig. 4 Camera screen coordination system viewed from a vie camera.

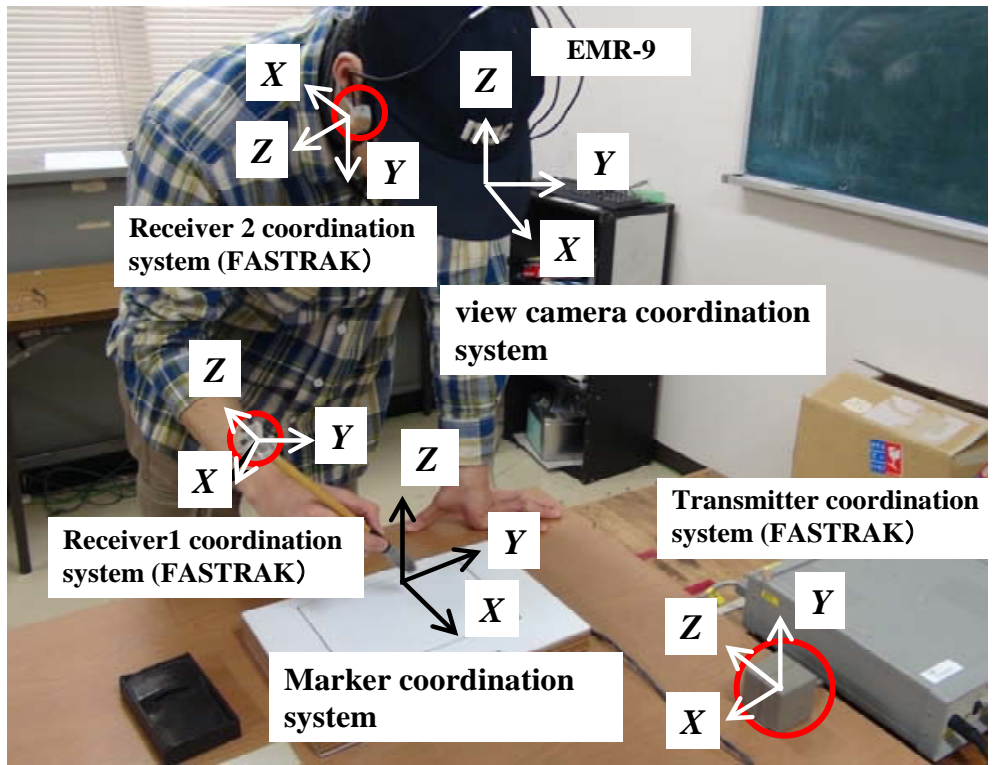

camera screen coordination system

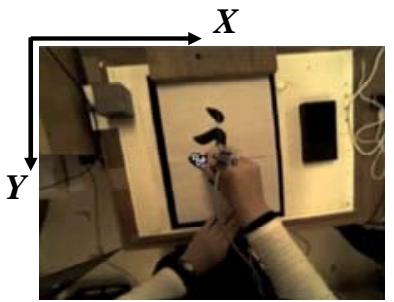

Fig. 5 Overview of measurement system and coordination system. 


\subsection{Calculation Method of Pen Tip Location}

The Receiver 1 of FASTRAK is attached so that the brush tip faces toward $-Z$. The brush location vector $\boldsymbol{V}_{p}$ is represented by Eq. (1), where $m$ is the length of brush.

$$
V_{p}=\left[\begin{array}{c}
0 \\
0 \\
-m \\
0
\end{array}\right]
$$

Next, the Receiver 1 coordination system is transformed to the transmitter coordination system using the location and posture data $\left(X, Y, Z, \varphi_{y}, \varphi_{p}, \varphi_{r}\right)$ from FASTRAK output. The transformation matrix ${ }^{t} \boldsymbol{T}_{r}$ is given by

$$
{ }^{t} \boldsymbol{T}_{r}=\boldsymbol{R}_{z} \boldsymbol{R}_{y} \boldsymbol{R}_{x} \boldsymbol{P}
$$

where the matrices $\boldsymbol{R}_{x}, \boldsymbol{R}_{y}, \boldsymbol{R}_{z}$ are the rotation matrices around $x$-, $y$-, and $z$-axis, respectively. The matrix $\boldsymbol{P}$ is the translation matrix. The matrices $\boldsymbol{R}_{x}, \boldsymbol{R}_{y}, \boldsymbol{R}_{z}$, and $\boldsymbol{P}$ are given by

$$
\begin{aligned}
& R_{x}= {\left[\begin{array}{cccc}
1 & 0 & 0 & 0 \\
0 & \cos \phi_{r} & -\sin \phi_{r} & 0 \\
0 & \sin \phi_{r} & \cos \phi_{r} & 0 \\
0 & 0 & 0 & 1
\end{array}\right] } \\
& R_{y}= {\left[\begin{array}{cccc}
\cos \phi_{p} & 0 & \sin \phi_{p} & 0 \\
0 & 1 & 0 & 0 \\
-\sin \phi_{p} & 0 & \cos \phi_{p} & 0 \\
0 & 0 & 0 & 1
\end{array}\right] } \\
& R_{z}= {\left[\begin{array}{cccc}
\cos \phi_{y} & -\sin \phi_{y} & 0 & 0 \\
\sin \phi_{y} & \cos \phi_{y} & 0 & 0 \\
0 & 0 & 1 & 0 \\
0 & 0 & 0 & 1
\end{array}\right] } \\
& P=\left[\begin{array}{llll}
1 & 0 & 0 & X \\
0 & 1 & 0 & Y \\
0 & 0 & 0 & Z \\
0 & 0 & 1 & 1
\end{array}\right]
\end{aligned}
$$

Thus, the brush tip vector $\boldsymbol{V}_{\boldsymbol{p} t}$ using the transmitter coordination system can be calculated using Eq. (7).

$$
V_{p t}={ }^{t} T_{r} V_{p}
$$

The value of $m$ was set to $280 \mathrm{~mm}$.

As the soft brush was used in the experiment, the flexure of the brush must be taken into account. Therefore, using the coordinates of the tail end of the brush and the brush tip, the brush location is described using a three-dimensional line. The working plane of the world coordination system is nearly the same with that of the transmitter coordination system with $y=$ $-27 \mathrm{~mm}$. The coordinate of intersection of the two planes was calculated to derive the actual contact location of the brush tip by taking the flexure of the brush into account. Thus, setting the coordinate of end of brush, the coordinate of brush tip, and the coordinate of the actual contact location of the brush to $\left(X_{1}, Y_{1}, Z_{1}\right),\left(X_{2}, Y_{2}, Z_{2}\right)$, and $\left(X_{w}, Y_{w}, Z_{w}\right)$, respectively, Eq.(8) is obtained.

$$
\frac{x-x_{1}}{x_{2}-x_{1}}=\frac{y-y_{1}}{y_{2}-y_{1}}=\frac{z-z_{1}}{z_{2}-z_{1}}
$$

Putting $Y=\left(y-y_{1}\right) /\left(y_{2}-y_{1}\right)$ and setting $y=-27, y_{1}$ $=Y_{1}$, and $y_{2}=Y_{2}$, Eq. (9) is obtained and $Y$ can be expressed using known values.

$$
Y=\frac{-27-Y_{1}}{Y_{2}-Y_{1}}
$$

From Eqs. (8) and (9), the following equations are obtained.

$$
\begin{aligned}
& X_{w}=Y\left(x_{2}-x_{1}\right)+x_{1} \\
& Z_{w}=Y\left(z_{2}-z_{1}\right)+z_{1}
\end{aligned}
$$

Thus, the actual contact location $\left(X_{w}, Y_{w}, Z_{w}\right)$ in the world coordination system is given by

$$
\left[\begin{array}{c}
X_{w} \\
Y_{w} \\
Z_{w}
\end{array}\right]=\left[\begin{array}{c}
Y\left(x_{2}-x_{1}\right)+X_{1} \\
-27 \\
Y\left(z_{2}-z_{1}\right)
\end{array}\right]
$$

\subsection{Calculation Method of Eye-Gaze Location after Correction of Binocular Parallax}

It is described how the eye-gaze point $(x, y)$ after the correction of binocular parallax is calculated using both right $\left(\left(x_{r}, y_{r}\right)\right)$ and left $\left(\left(x_{l}, y_{l}\right)\right)$ eye marks output from EMR-9. As shown in Fig. 6, the right or the left eye mark corresponds to the intersection of the eye-gaze vector that connects the eye-gaze point and the right or left eye and the calibration plane. The 
camera screen coordination system is shown in Fig. 6.

The eye-gaze point $(x, y)$ after the correction of binocular parallax can be calculated by deriving the intersection of the eye-gaze vector that connects the eye-gaze point and the view camera and the calibration plane. As shown in Fig. 6, the eye-gaze points $(x, y)$ after the correction of binocular parallax appears on the perpendicular bisector of the line connecting right and left eye marks. It must be noted that the right $\left(x_{r}, y_{r}\right)$ and left $\left(x_{l}, y_{l}\right)$ eye marks corresponds with the eye-gaze point $(x, y)$ after the correction of binocular parallax on the camera screen coordination system when the eye-gaze point is on the calibration plane. As shown in Figs. 7 and 8, there are two cases, that is, when the gaze-point is beyond the calibration plane, and when the gaze-point is before the calibration plane.

First, the calculation method of the eye-gaze point $(x, y)$ after the correction of binocular parallax is described when the gaze-point is beyond the calibration plane. The upper view of Fig. 7 (when Fig. 7 is viewed from the upper and perpendicular direction) is shown in Fig. 9. The side view of Fig. 7 (when Fig. 7 is viewed from the horizontal direction) is shown in Fig. 10. From Fig. 9, the binocular parallax $\Delta v$ is given by

$$
\Delta v: w=L-l: L
$$

Thus, $L$ is given by Eq. (14).

$$
L=\frac{w t}{w-\Delta v}
$$

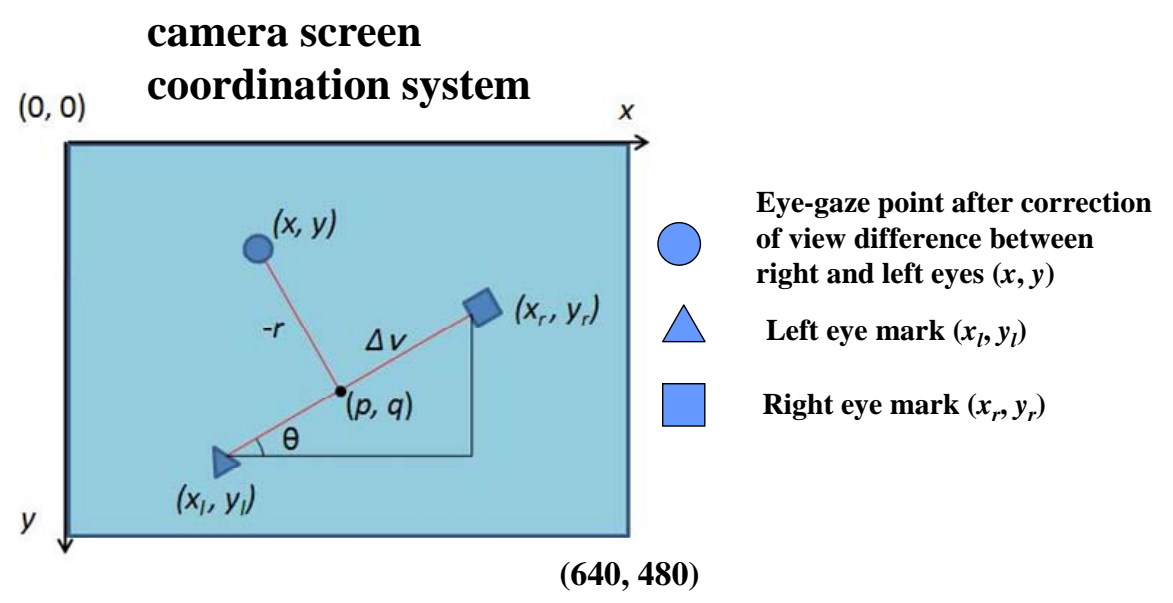

Fig. 6 Relationship between eye-gaze point and right or left eye mark in camera screen coordination system.

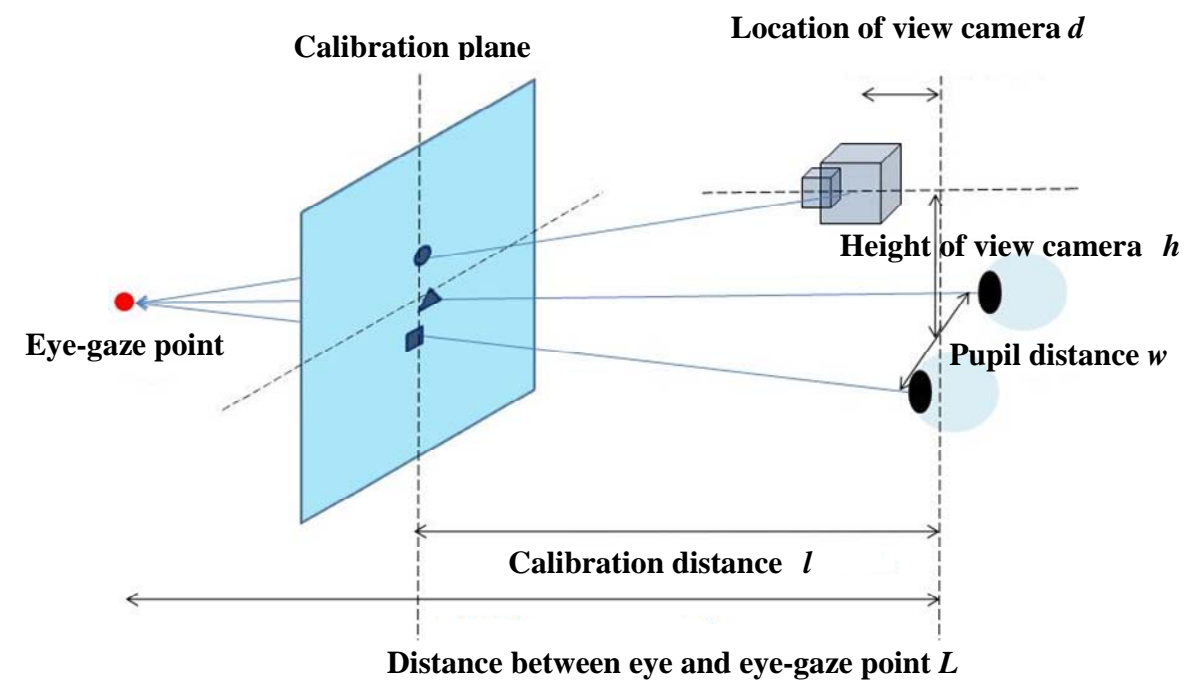

Fig. 7 Geometric relationship when the gaze-point is beyond the calibration plane. 

Measurement of Eye-gaze and Hand Location-

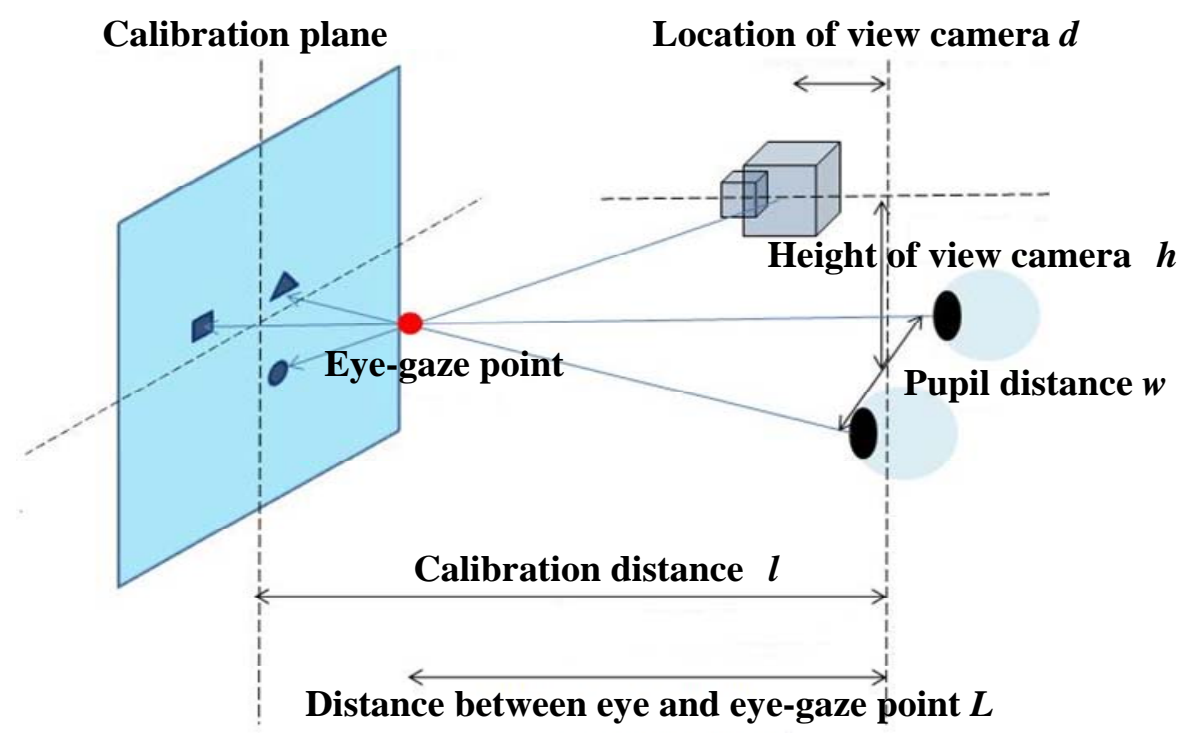

Fig. 8 Geometric relationship when the gaze-point is before the calibration plane.

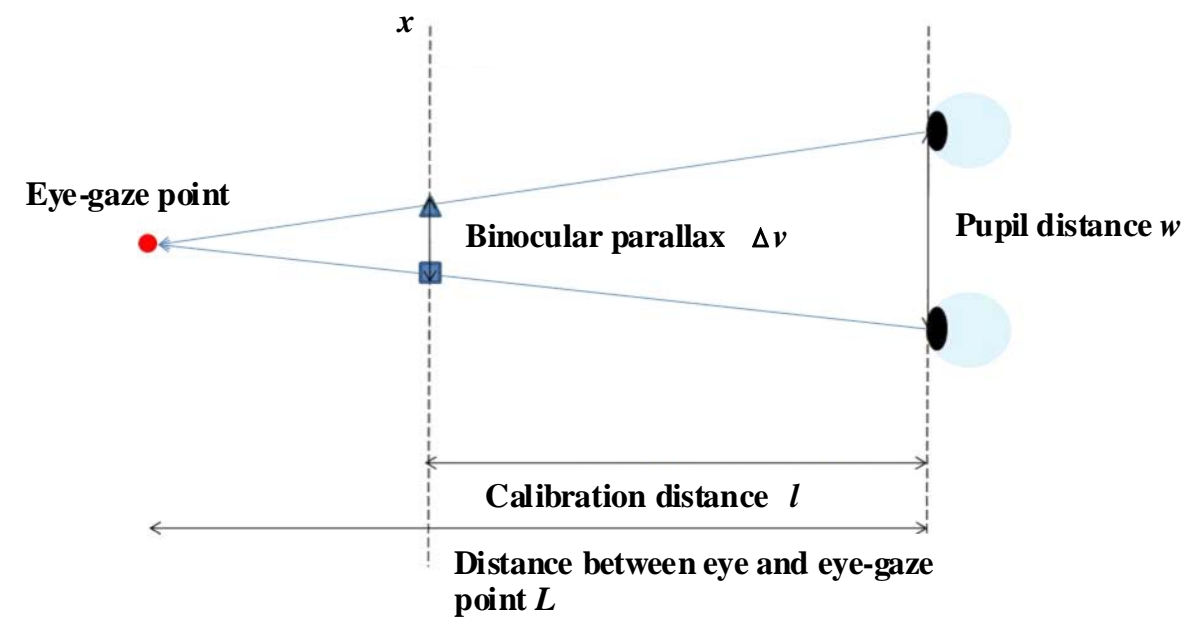

Fig. 9 Geometry when Fig. 7 is viewed from the upper and perpendicular direction.

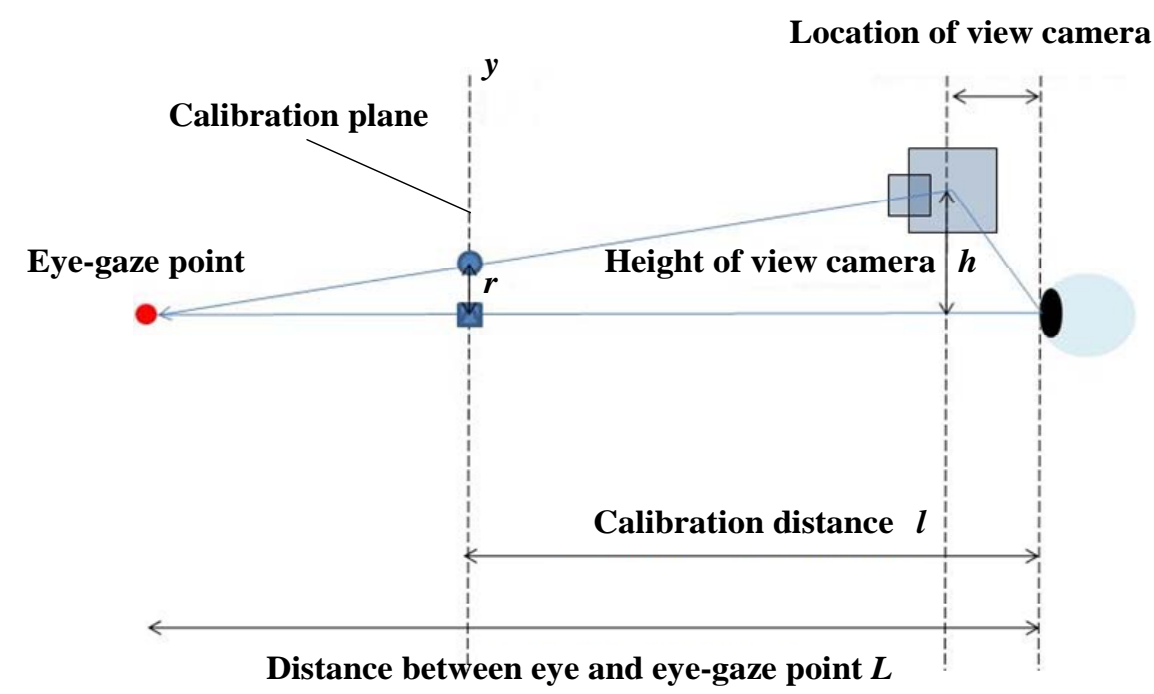

Fig. 10 Geometry when Fig. 7 is viewed from the horizontal direction. 
The binocular parallax $\Delta v$ can be represented by

$$
\Delta v=\sqrt{\Delta x^{2}+\Delta y^{2}}
$$

From Fig. 10, Eq. (16) is obtained.

$$
r: h=L-l: L-d
$$

Solving Eq. (16) with respect to $r$, Eq. (17) is obtained.

$$
r=\frac{L-l}{L-d} h
$$

Using Eq. (15) and Eq. (17), Eq. (18) is obtained.

$$
r=\frac{h l \Delta v}{w(l-d)+d \Delta v}
$$

Setting the coordinate of the middle point between the right and the left eye mark $(p, q)$, the eye-gaze point $(x, y)$ after correction of binocular parallax is given by Eqs. (19) and (20).

$$
\begin{aligned}
& x=p+r \sin \theta \\
& y=q+r \cos \theta
\end{aligned}
$$

\subsection{Calculation Method for Eye-Hand Coordination} System-Transformation of Eye-Gaze Location after Correcting Binocular Parallax to Transmitter Coordination System of Brush Tip Location-

The method to transform the eye-gaze point after correcting the binocular parallax $\left(x_{c s}, y_{c s}, z_{c s}\right)$ to the transmitter coordination system $\left(x_{t}, y_{t}, z_{t}\right)$ by which the brush tip location is expressed.

The eye-gaze point in the camera screen coordination system is transformed to the eye-gaze point according to the following procedure. Using the inner-camera parameter ${ }^{c s} \boldsymbol{T}_{c}$ and outer-camera parameter ${ }^{c} \boldsymbol{T}_{m}$, the transformation matrix from the marker coordination system to the camera screen coordination system ${ }^{c s} \boldsymbol{T}_{m}$ is calculated.

$$
{ }^{c s} \boldsymbol{T}_{m}={ }^{c s} \boldsymbol{T}_{c}{ }^{c} \boldsymbol{T}_{m}
$$

where ${ }^{c s} \boldsymbol{T}_{m}$ is a $3 \times 4$ matrix. When transforming the marker coordination system to the camera screen coordination system, the information on $Z$-axis is missing. Therefore, the 3-rd row of ${ }^{c s} \boldsymbol{T}_{m}$ was deleted. Calculation of the inverse matrix ${ }^{c s} \boldsymbol{T}_{m}{ }^{-1}$ enabled us to obtain the eye-gaze point on the $x-y$ plane in the marker coordination system as follows.

$$
\left[\begin{array}{l}
x \\
y \\
z
\end{array}\right]={ }^{c s} T_{m}^{-1}\left[\begin{array}{c}
x_{c s} \\
y_{c s} \\
1
\end{array}\right]
$$

Normalizing the values of the marker coordination system obtained by Eq. (22) and adding $z_{m}=0$ to the vector given by Eq. (22) leads to Eq. (23).

$$
\left[\begin{array}{c}
x_{m} \\
y_{m} \\
z_{m} \\
1
\end{array}\right]={ }^{c s} T_{m}^{-1}\left[\begin{array}{c}
x / s \\
y / s \\
0 \\
1
\end{array}\right]
$$

The eye-gaze point in the marker coordination system given by Eq. (23) is transformed into the transmitter coordination system using the transformation matrix ${ }^{t} \boldsymbol{T}_{m}$ given by Eq. (24). The transformation matrix ${ }^{t} \boldsymbol{T}_{m}$ is represented as follows. As the center of AR marker is set to the coordinate $(200,-27,150)$ of the transmitter coordination system, the transformation matrix ${ }^{t} \boldsymbol{T}_{m}$ can be defined as the following matrix that moved $200 \mathrm{~mm}$ along $x$-axis, $-27 \mathrm{~mm}$ along $y$-axis, and $150 \mathrm{~mm}$ along $z$-axis, and rotated 90 degrees around $x$-axis.

$$
{ }^{t} T_{m}=\left[\begin{array}{cccc}
1 & 0 & 0 & 200 \\
0 & \cos 90^{\circ} & \sin 90^{\circ} & -27 \\
0 & -\sin 90^{\circ} & \cos 90^{\circ} & 150 \\
0 & 0 & 0 & 1
\end{array}\right]
$$

The transmitter coordination system is transformed into the Receiver 2 coordination system attached to EMR-9 in Fig. 5. The transformation matrix ${ }^{r 2} \boldsymbol{T}_{t}$ can be calculated as the inverse matrix that transforms the transmitter coordination system to the receiver coordination system. On the basis of the transformation above, the eye-gaze point $\left(x_{c s}, y_{c s}\right)$ in the camera screen coordination system can be transformed into the eye-gaze point $\left(x_{r 2}, y_{r 2}, z_{r 2}\right)$ in the Receiver 2 coordination system. Thus, the eye-gaze point in the Receiver 2 coordination system $\left(x_{r 2}, y_{r 2}\right.$, $z_{r 2}$ ) can be represented by 

Measurement of Eye-gaze and Hand Location-

$$
\left[\begin{array}{c}
x_{r 2} \\
y_{r 2} \\
z_{r 2} \\
1
\end{array}\right]={ }^{r 2} T_{t}^{t} T_{m}\left[\begin{array}{c}
x_{m} \\
y_{m} \\
z_{m} \\
1
\end{array}\right]
$$

As both view camera and Receiver 2 are attached (fixed) to EMR-9, the location and the posture between these two systems does not change even if the head location of the participant changes. Therefore, the transformation matrix that transforms the eye-gaze point in the camera screen coordination system into the eye-gaze point in the Receiver 2 coordination system is unchanged. The location of the Receiver 2 changes according to the head movement of the participant. The change of the eye-gaze point $\left(x_{r 2}, y_{r 2}\right.$, $z_{r 2}$ ) due to head movement is transformed into the eye-gaze point in the transmitter coordination system. Setting the transformation matrix to ${ }^{t} \boldsymbol{T}_{r 2}$, the eye-gaze point $\left(x_{t}, y_{t}, z_{t}\right)$ in the transmitter coordination system is given by

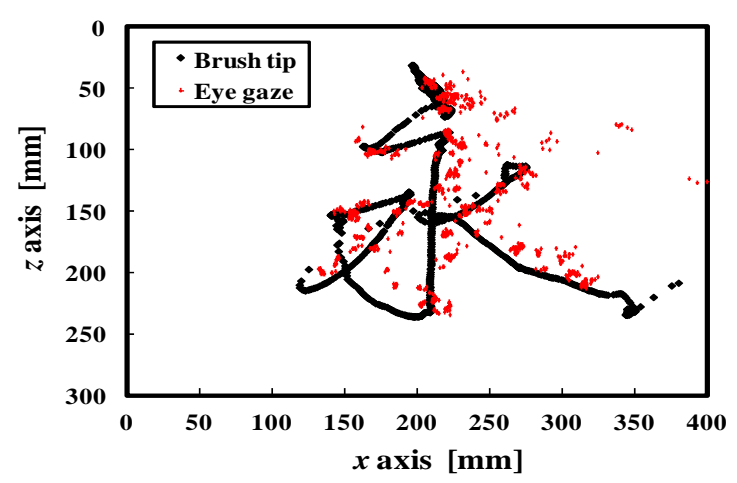

(a) $「$ 永」(Forever)

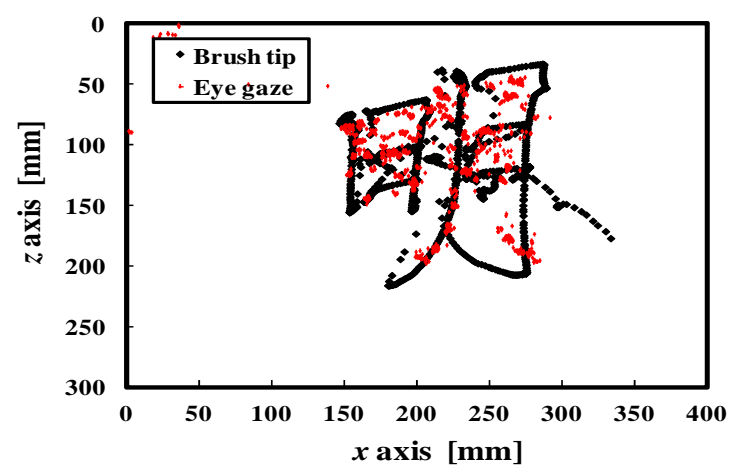

(c)「明」(Bright)

$$
\left[\begin{array}{c}
x_{t} \\
y_{t} \\
z_{t} \\
1
\end{array}\right]={ }^{t} T_{r 2}\left[\begin{array}{c}
x_{r 2} \\
y_{r 2} \\
z_{r 2} \\
1
\end{array}\right]
$$

By moving $\left(x_{t}, y_{t}, z_{t}\right)$ along $Y$-axis by $-27 \mathrm{~mm},\left(x_{t}, y_{t}\right.$, $z_{t}$ ) corresponds with the world coordination system of the location of the brush tip.

\section{Example of Application of the Measurement System to Skill Evaluation in Calligraphy}

The measurement was carried out during the calligraphic task for both novice and expert. The developed real-time eye-hand coordination system was used to measure eye movements and brush tip location during the calligraphic task.

Examples of the positions of each brush tip stroke and eye-gaze are plotted shown in Fig. 11 (skilled participant) and Fig. 12 (non-skilled participant). The

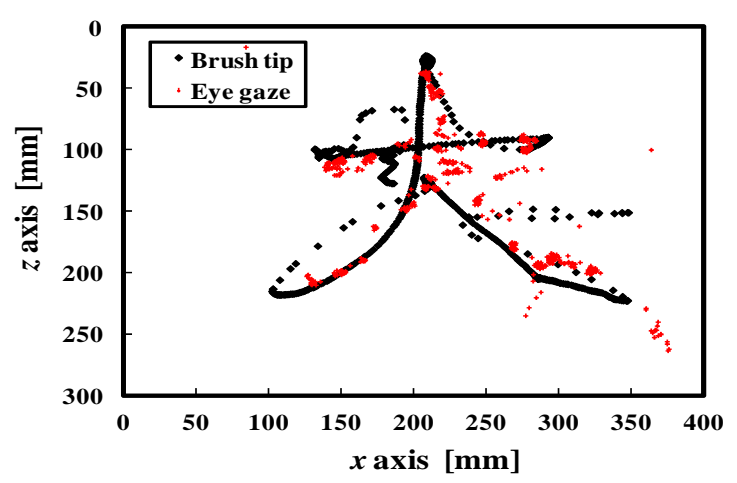

(b)「大」(Big)

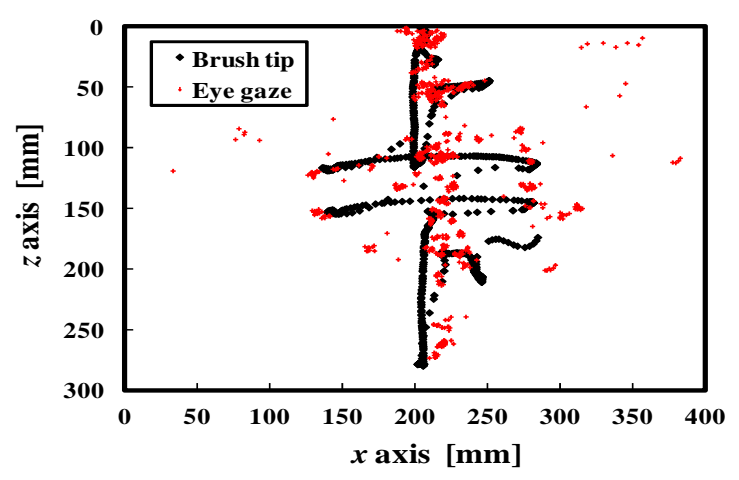

(d)「上下」(ups and downs)

Fig. 11 Trajectory of brush tip and eye-gaze point (skilled participant). 

Measurement of Eye-gaze and Hand Location-

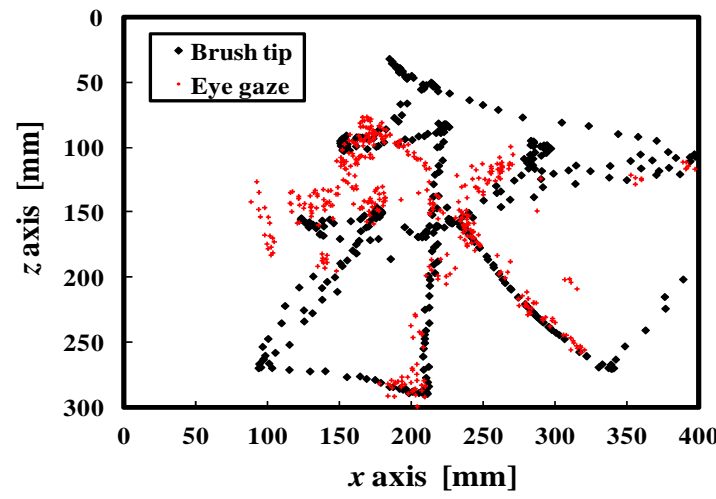

(a)「永」(Forever)

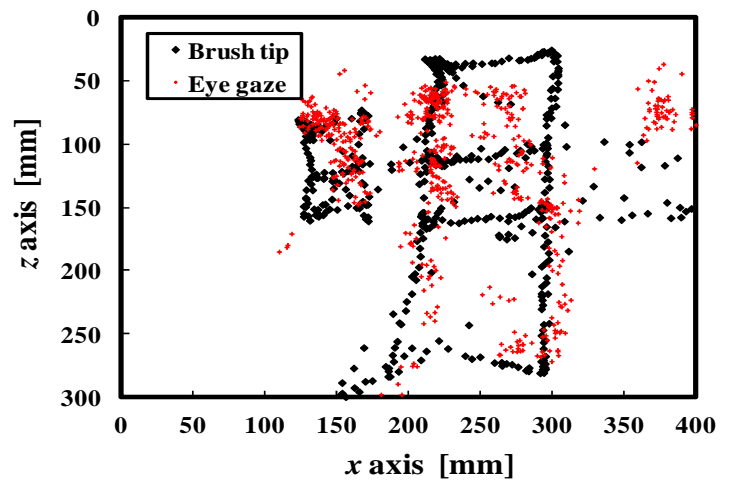

(c)「明」(Bright)

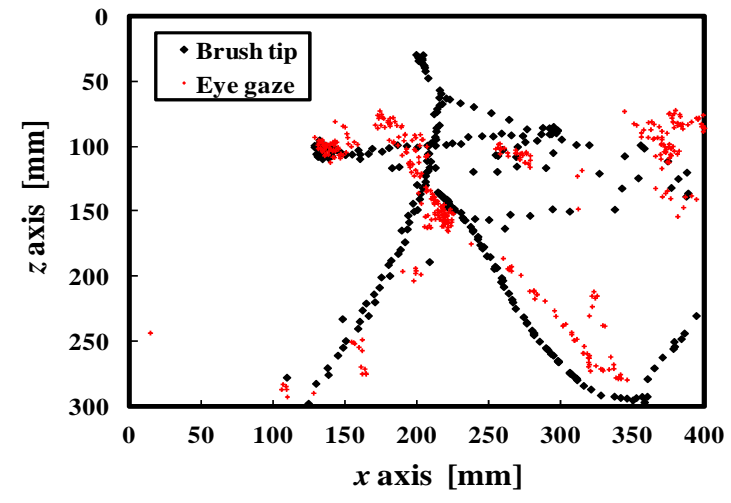

(b)「大」(Big)

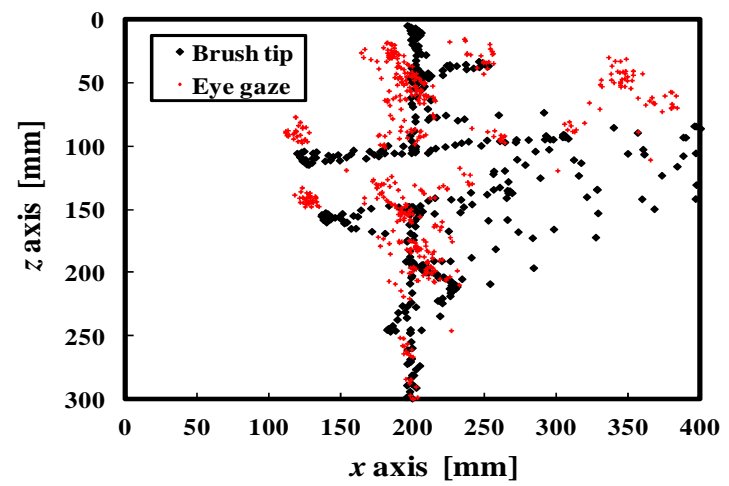

(d)「上下」(ups and downs)

Fig. 12 Trajectory of brush tip and eye-gaze point (non-skilled participant).

Skilled participant

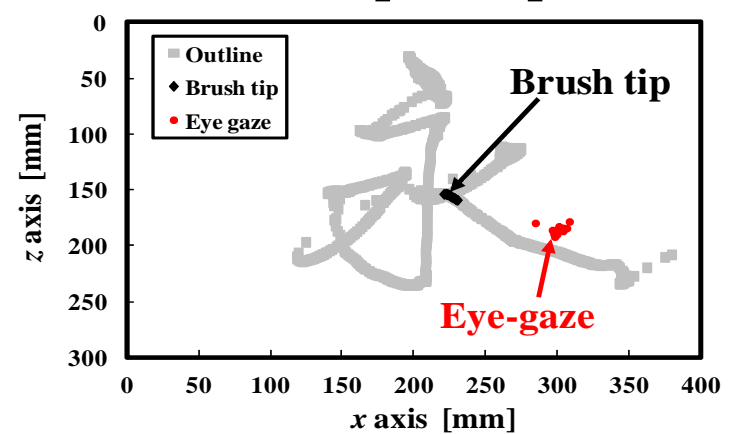

Non-skilled participant

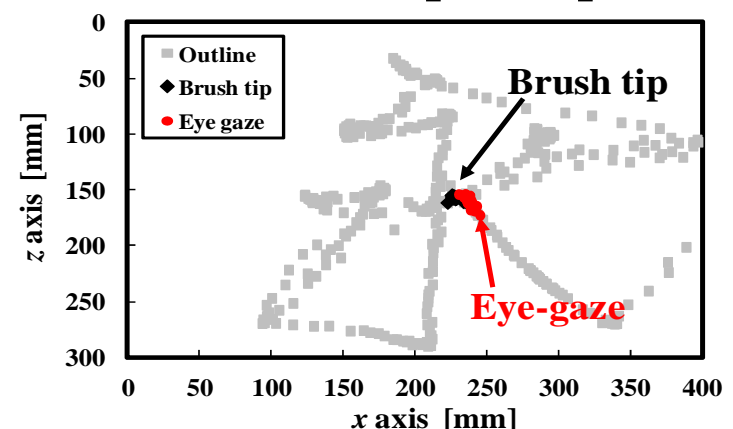

Fig. 13 Comparison of brush tip and eye-gaze locations between skilled and non-skilled participant (5-th stroke of 「永」 (forever)).

brush tip and eye-gaze locations when writing the 5-th stroke of Kanji character "forever" are compared between a skilled and a non-skilled participant in Fig. 13. As for the skilled participant, the brush tip location is distant from the eye-gaze location. As far as the novice is concerned, the brush tip exists at nearly the same location with the eye-gaze location. On the basis of the finding that the eye movement is preceding the brush tip for the skilled participant, it is possible that such a property might be one of the important skills necessary for acquiring skills in calligraphy.

In such a way, the proposed method for real-time measurement system of eye-hand coordination is promising for clarifying the process of skill acquisition in skilled movement such as calligraphy. In future research, such a skilled element pointed out 
in the demonstrative experiment must be verified by a systematic experiment using the developed real-time measurement system of eye-hand coordination.

\section{Conclusions}

The aim of this stuffy was to propose a method for measuring eye-hand coordination which consisted of (1) calculation of pen tip location, (2) calculation of eye-gaze location after correction of binocular parallax, and (3) transformation of eye-gaze location to the same coordination system with pen tip location so as to enable us to view simultaneously the eye-gaze and the brush tip locations. Calligraphy was selected as an application of the proposed measurement system of eye-hand coordination. As far as our experiment is concerned, it has been suggested that experts might be different from novices in the eye movement preceding brush tip, and such a property might be an important skilled element for acquiring a higher skill.

\section{References}

[1] Vickers, J. 1995. "Gaze Control in Basketball Foul Shooting." In Eye Movement Research: Mechanisms, Processes, and Applications, edited by Findlay, J., Walker, R., and Kentridge, R. Amesterdam: Elsevier, 527-41.

[2] Murata, A., and Moriwaka, M. 2009. "Skill of Eye-Hand Coordination in Calligraphy-Difference of Skill of Eye-Hand Coordination between Expert and Novice-." In Proceedings of 5th International Workshop on Computational Intelligence \& Applications, 316-9.

[3] Murata, A., Inoue, K., and Moriwaka, M. 2011. "Real-Time Measurement System of Eye-Hand Coordination in Calligraphy." In Proceedings of SICE2011, 2696-701.

[4] Murata, A., Ioue, K., Hayami, T., and Moriwaka, M. 2012. "Real-Time Measuring System of Eye-Gaze Location and Writing Pressure in Calligraphy." In Proceedings of AHFE2012, 8290-9.

[5] Sano, T., and Ukida, H. 2010. "Measurement of Handwriting Skills for Japanese Calligraphy." In Proceedings of Instrumentation and Measurement Technology Conference, 1112-5. 